\title{
Case Report \\ Acute Fulminant Eclampsia with Cardiopulmonary arrest, Pulmonary Oedema and PRES: Maternal and Fetal Outcome
}

\author{
Papa Dasari ${ }^{*}$ (ID), M. Nithin \\ Department of OBGY, JIPMER, Puducherry, India
}

\section{ARTICLE INFO}

\section{Article History}

Received 13 May 2021

Accepted 26 August 2021

Keywords

Fulminant eclampsia

cardiac arrest

PRES

pulmonary oedema

\begin{abstract}
Complicated eclampsia resulting in cardiac arrest and subsequent fetal and maternal survival is rare. A 25-year-old primigravida was referred after initial resuscitation for cardiac arrest following a maternal collapse during pregnancy due to eclampsia at 36 weeks of pregnancy. The diagnosis at referral was antepartum eclampsia, pulmonary oedema and intrauterine fetal death. Re-evaluation revealed presence of fetal cardiac activity on ultrasound and an immediate emergency Caesarean Section was carried out and a live baby with APGAR of 3/10 was delivered and cared for in neonatal intensive care unit after intubation and positive pressure ventilation. Mother was managed in obstetric intensive care unit and was on ventilator for $72 \mathrm{~h}$. She was treated for pulmonary oedema and persistent hypertensive crisis requiring multiple antihypertensives including nitroglycerine. Her echocardiogram showed global hypokinesia and left ventricular ejection fraction of $45 \%$ on day 1 which later improved to normal by day 15. MRI brain showed features of posterior reversible encephalopathy syndrome. She was given levitricetam for seizure prophylaxis. In addition, she required treatment for aspiration pneumonia. She was discharged after 16 days on two antihypertensive drugs along with her baby in good health. Timely resuscitation following cardiac arrest and transport to a tertiary care centre and control of hypertensive crisis with multidisciplinary care were the life-saving factors in this case.
\end{abstract}

(C) 2021 First Affiliated Hospital of Zhengzhou University. Publishing services by Atlantis Press International B.V. This is an open access article distributed under the CC BY-NC 4.0 license (http://creativecommons.org/licenses/by-nc/4.0/).

\section{INTRODUCTION}

Hypertension during pregnancy is the leading cause of maternal morbidity and mortality. It has been recognised that the disease can be predicted and prevented to certain extent by evidence based interventions. Hypertension is a multi-system disease and the unique complication during pregnancy is eclampsia. Maternal mortality due to eclampsia is variously reported as $10-15 \%$ [1] in developed countries and 43\% in India [2].

Eclampsia can be prevented as premonitory symptoms and signs precede in majority of the cases and timely administration of prophylactic magnesium sulphate therapy is a proven intervention to prevent eclampsia. Eclampsia has a rapid onset and can have a fulminant course with complications including maternal collapse and death.

Survival rate after Cardiopulmonary Resuscitation (CPR) is only $50 \%$ in literature. The most important factors that influence survival after CPR are Return of Spontaneous Circulation (ROSC) and the type of institutional care. Mortality has been observed to be lower in tertiary care hospitals [3]. This case report illustrates the onset of fulminant eclampsia and subsequent events of cardiopulmonary arrest, pulmonary oedema, aspiration and Posterior Reversible Encephalopathy Syndrome (PRES) with maternal and fetal outcome with intensive care therapy.

Corresponding author. Email: dasaripapa@gmail.com

Peer review under responsibility of the First Affiliated Hospital of Zhengzhou University
CASE: Mrs S, a 25 year old primigravida married for 5 years, belonging to low socioeconomic status, was shifted to our Emergency services at $36+2$ days in morbid state after intubation and initial resuscitation at a district hospital. She was accompanied by the anaesthetist of the medical centre who resuscitated her following maternal collapse and cardiac arrest.

She was hospitalised in the medical centre 3 days ago for severe pre-eclampsia and offered oral labetalol $100 \mathrm{mg}$ twice daily and received antenatal corticosteroids for fetal lung maturity. She had seizures in the antenatal ward in the presence of doctors and nurses and collapsed with pink frothy sputum coming from mouth. She was immediately transferred to eclampsia room and had two more episodes of convulsions and sustained cardiac arrest. She was given two bolus doses of adrenaline and $200 \mathrm{mg}$ of intravenous hydrocortisone. She also received injections of phenytoin and midazolam after successful intubation. However magnesium sulphate was withheld due to suspected pulmonary oedema. The referral diagnosis was antepartum ecalmpsia with pulmonary oedema and intrauterine death. She was admitted to the obstetric Intensive Care Unit (ICU) and was immediately treated by the obstetric and anaesthetic consultants on call.

An in-depth review of her medical history after admission indicated that she has been married for 5 years and she had naturally conceived after investigations for infertility. She confirmed her pregnancy after 2 months of amenorrhea and registered under State Health Mission in a near by primary care centre. She had regular antenatal check-ups and her antenatal records indicated 
normal Blood Pressure (BP) recordings and normal weight gain. Her scan for screening fetal anomalies was normal. She has been immunised for tetanus toxoid and her antenatal visit record at 27 weeks showed haemoglobin of $8.6 \mathrm{gm} \%$. Her urine protein was ++ at this visit and there was no documentation of BP. She was treated with iron sucrose at the same centre and her haemoglobin was $9.5 \mathrm{gm} \%$ during the subsequent antenatal visit at 34 weeks and normal BP was documented.

There was no significant past and family history of communicable or non-communicable disorders. Her haematological investigations were within normal limits at the time of hospitalisation at the District hospital (Secondary Care) where she has been hospitalised and this was a sudden unexpected event.

On examination, she appeared bloated up, pupils were reacting to light and her Glasgow Coma Scale was E1Vt M1. Pulse rate was $140 / \mathrm{min}$ regular, $\mathrm{BP} 170 / 108 \mathrm{mmHg} \mathrm{SpO}_{2} 75 \%$ with $10 \mathrm{~L}$ of Oxygen $/ \mathrm{min}$. There was $350 \mathrm{ml}$ blood stained urine in Urobag. Respiratory rate was $42 / \mathrm{min}$ and there were bilateral coarse crepitations. Cardiovascular system examination showed tachycardia and no cardiac murmurs were heard. Quick abdominal examination revealed gross oedema and term size relaxed uterus with single fetus in cephalic presentation. Fetal heart was not localised on initial examination but her ultrasound revealed fetal bradycardia $(<100$ beats $/ \mathrm{min})$ and the estimated fetal weight was $2.2 \mathrm{~kg}$. Per vaginal examination showed unfavourable cervix and fetal station was above brim. A decision was made to do emergency Lower Segment Caesarean Section (LSCS) with high risk consent. A provisional diagnosis of antepartum eclampsia with pulmonary oedema and suspected aspiration with fetal bradycardia was made. She was given $20 \mathrm{mg}$ of furosemide intravenously and $9 \mathrm{mg}$ of morphine intramuscularly and $10 \mathrm{~L}$ of Oxygen $/ \mathrm{min}$. Arterial blood gas analysis showed $\mathrm{pH}$ - of 7.22, $\mathrm{pO}_{2}-48.6 \mathrm{pa}, \mathrm{SO}_{2}-73.5 \%$. Prophylactic antibiotics were administered. Emergency caesarean section was performed under general anaesthesia and her BP was maintained at a mean arterial pressure of $100-110 \mathrm{mmHg}$. A live preterm baby was born with APGAR of 3 at $1 \mathrm{~min}$ and 5 at $5 \mathrm{~min}$. The baby was shifted to neonatal intensive care unit after intubation and resuscitation and the birth weight was $2.2 \mathrm{~kg}$.

Her pulse rate was $92 / \mathrm{min}$, BP was $165 / 89 \mathrm{mmHg}$ at the beginning of surgery and end operative pulse rate was $80 / \mathrm{min}$ and BP was 130/90 $\mathrm{mmHg}$ and estimated blood loss was less than $500 \mathrm{ml}$. She received only $300 \mathrm{ml}$ of crystalloids during surgery.
Her oxygen saturation varied between $80 \%$ and $90 \%$ during LSCS. Post-operatively she remained in ICU with ventilator support. Levetiracetam injections were started for prevention of further convulsions. Morphine and midazolam infusions were maintained.

Her immediate post-operative haemoglobin was $8.5 \mathrm{gm} \%$ and platelet count was 1.5 lakhs but haematuria persisted. She therefore received fresh frozen plasma $25 \mathrm{ml} / \mathrm{kg}$ body weight 8th hourly and intravenous fluids at $20 \mathrm{ml} / \mathrm{h}$ and intravenous furosemide $40 \mathrm{mg} 12$ hourly. She was started on intravenous piperacillin $4.5 \mathrm{~g} 12$ hourly, amikacin $375 \mathrm{mg}$ once daily and metronidazole $500 \mathrm{mg} 8$ hourly. Bedside X-ray chest showed features of pulmonary oedema and aspiration especially on right side (Figure 1A). Echocardiogram (ECHO) showed left ventricular diastolic diameter of $4.4 \mathrm{~cm}$, global hypokinesia of left ventricle with ejection fraction of $45 \%$. She was started on tablet enalapril $2.5 \mathrm{mg}$ through ryles tube. Hypertensive crisis was managed by Nitroglycerine (NTG) infusion and dose titration done to keep the Mean Arterial Pressure (MAP) at 90-100 mmHg. Fundus examination showed grade 2 hypertensive retinopathy and there was no papilledema. Magnesium sulphate $1 \mathrm{~g} / \mathrm{h}$ infusion was started on post-operative day 1 . After $48 \mathrm{~h}$ she regained consciousness after stopping Midazolam infusion. Hypertensive crisis recurred $(160 / 117 \mathrm{mmHg})$ despite NTG infusion. Intravenous labetalol $20 \mathrm{mg}$ was given and she was sedated with midazolam again as she was restless due to the endotracheal tube block and leakage which was identified and rectified. Abdominal gaseous distension was managed by inserting dulcolax rectal pessary. Nitroglycerine was ceased after BP stabilised at 120/70 mmHg. She subsequently received oral. Amlodipine $5 \mathrm{mg} 12$ hourly, enalapril $2.5 \mathrm{mg}$ 12 hourly and oral aldactone (Spiranolactone) $25 \mathrm{mg}$ once daily through ryles tube. She continued to receive intravenous hydrocortisone $100 \mathrm{mg}$ once daily for 3 days. She was extubated on third post-operative day and continued on nasal oxygen 4-6 L/min and Furacort (Formoterol Fumarate Dihydrate) nebulisation 8 hourly for 1 week along with chest physiotherapy. Her follow-up ECHO on third day of intensive care stay showed an improved ejection fraction (52\%) and mild global hypokinesia. Chest X-rays repeated on third and sixth day of intensive care stay (Figure 1B and 1C) showed improvement in lung fields. CT brain done on fifth post-operative day (Figure 2) showed features of frontal lobe and occipital lobe oedema suggestive of PRES which was confirmed by MRI brain performed 2 days later (Figure 3).
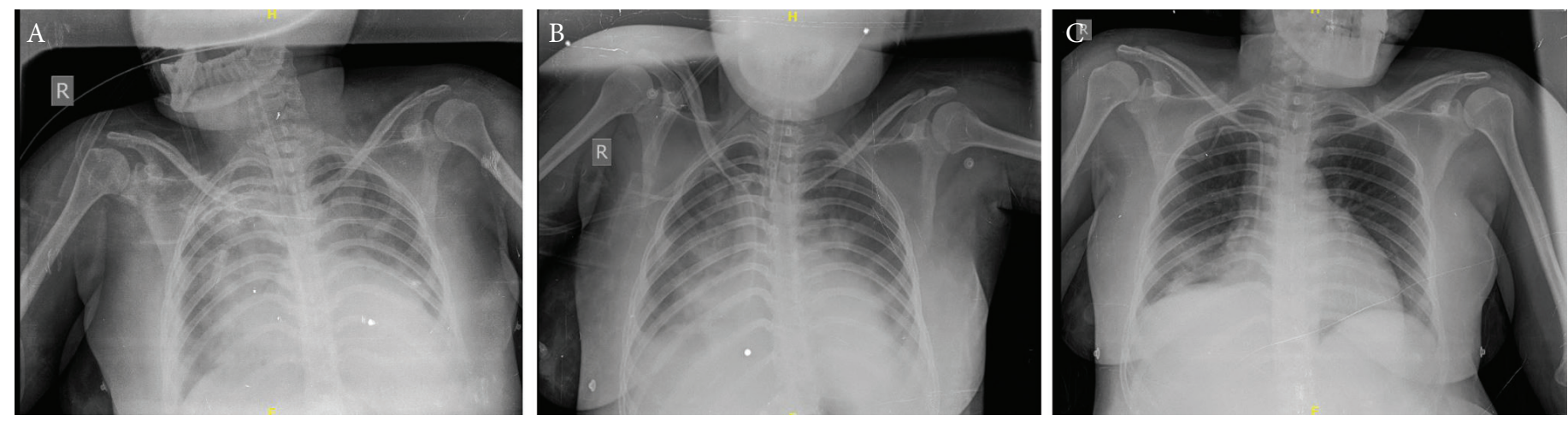

Figure 1 (A) X-ray chest P/A day of admission shows ground glass appearance and early pneumonitis changes suggestive of pulmonary oedema and aspiration. (B) X-ray chest P/A; day 3 shows patches of lower lobe pneumonia right lung (following aspiration). (C) X-ray chest P/A; day 6 normal lungs. 


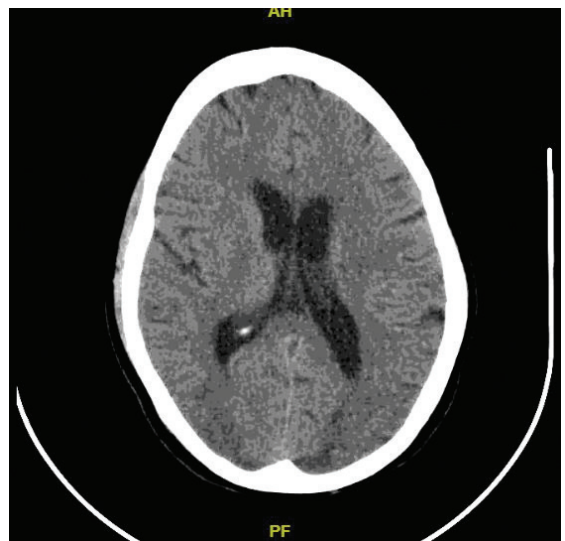

Figure 2 CECT brain: hyperdensities in frontal lobe and occipital lobe suggestive of PRES.

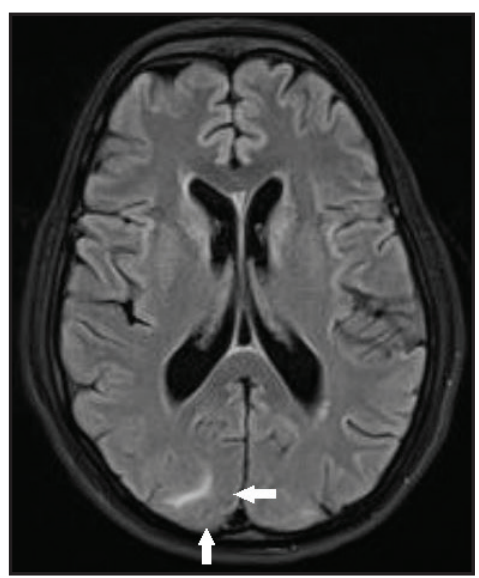

Figure 3 MRI brain: white matter vasogenic oedema in occipital lobe confirmation of PRES.

Amlodipine and aldactone (Spiranolactone) were stopped and she was advised to continue on oral envas and metaprolol $12.5 \mathrm{mg}$ was added. Her BP was controlled and maintained at $126 / 80 \mathrm{mmHg}$. Echocardiogram on 15 th post-operative day revealed concentric left ventricular hypertrophy and a normal ejection fraction. She recovered well and breastfed the baby from the seventh post-natal day and both mother and baby were discharged in good health on the 16th post-natal day. She was advised to continue oral metaprolol and oral envas, tab. levetiracetam along with haematinics and review after 2 weeks. The final diagnosis was acute fulminant eclampsia PRES, pulmonary oedema after a successful resuscitation post-cardiac arrest status.

\section{DISCUSSION}

Mortality in eclampsia is due to the complications like aspiration, cerebrovascular accident, pulmonary oedema and abruption. Sudden cardiac arrest is a rare event and is diagnosed by the absence of pulse or heart beat on auscultation within $1 \mathrm{~h}$ of convulsive episode [4] Cardiopulmonary arrest in pregnancy is rare occurring in one in 30,000 pregnancies and only 50\% survival [3]. Pulmonary oedema occurs in $2.9 \%$ of all severe pre-eclampsia and eclampsia [5] and 30\% occur in the antepartum period [6].
Posterior reversible encephalopathy syndrome (PRES) occurs in more than $90 \%$ of women with eclampsia [7] and it can be predicted in young women with hypertensive crisis, thrombocytopenia and proteinuria [8]. The clinical manifestations of PRES include headache, altered mental status, loss of consciousness and seizures. Typical findings on MRI are vasogenic oedema with $\mathrm{T}_{2}$ hyperintensities in the posterior subcortical and white matter of occipital lobes.

Mrs S had severe pre-eclampsia which developed 3 days prior to the onset of eclampsia. Despite her BP being controlled with single anti-hypertensive drug she developed sudden onset eclampsia without any premonitory symptoms. The cardiovascular systemic effects manifesting in the cerebral circulation include PRES and Reversible Cerebral Vasoconstriction Syndrome (RCVS) and both of these have been reported to coexist in one woman [9] and we posit that this may be the case of Mrs S.

Maternal outcome was good in an obese postpartum woman who sustained cardiac arrest after a convulsion. ROSC resulted within 3 min of CPR in spite of difficulties in resuscitation due to obesity [10]. Sudden cardiac arrest soon after convulsions can be due to magnesium sulphate toxicity $[11,12]$ or direct cardiac dysfunction such as atrial fibrillation, ventricular fibrillation [13]. These were not documented in Mrs S at or immediately after cardiac arrest.

Pulmonary oedema complicates $2.9 \%$ of women with pre-eclampsia and it is a life threatening complication if not recognised and treated [5]. This can be fulminant and associated with hypertensive crisis [14]. In Mrs S, pulmonary oedema was suspected initially as she collapsed and had pink frothy sputum and hence magnesium sulphate was withheld. There were no risk factors for pulmonary oedema except pre-eclampsia for her. Pulmonary oedema as well as aspiration were complications in her case and these were identified on X-ray and were managed appropriately.

Central apnoea can occur immediately after convulsions and Mrs S had apnoea as well as cardiac arrest and was revived immediately as the incident happened during day time working hours in the presence of multiple well trained health professionals. Van der Lende et al. [15] reported regarding central apnoea that occurred in the immediate post-ictal state before sustaining cardiac arrest.

\section{CONCLUSION}

- Complications of eclampsia can be fulminant and lead to acute cardiac arrest which is a rare event during pregnancy.

- In situation of maternal cardiac arrest fetal heart may not be heard on routine clinical examination and therefore absence of fetal heart by clinical examination needs confirmation by ultrasound.

- Immediate termination of pregnancy by caesarean section after first aid is helpful for efficient resuscitative measures to be effective.

- Women with complicated eclampsia need to be transported to tertiary care accompanied by a suitable trained health care professional (such as a doctor) and not paramedical workers without appropriate training and expertise.

- Timely CPR and ICU management can save maternal and fetal life when cardiac arrest occurs during pregnancy. 


\section{CONFLICTS OF INTEREST}

The authors declare they have no conflicts of interest.

\section{AUTHORS' CONTRIBUTION}

Papa Dasari contributed to case management supervision and manuscript preparation. M. Nithin contributed to case management in ICU under supervision and providing images and investigation reports.

\section{REFERENCES}

[1] Saleem S, McClure EM, Goudar SS, Patel A, Esamai F, Garces A, et al. A prospective study of maternal, fetal and neonatal deaths in low-and middle-income countries. Bull World Health Organ $2014 ; 92 ; 605-12$

[2] Das R, Biswas S. Eclapmsia: the major cause of maternal mortality in eastern India. Ethiop J Health Sci 2015;25;111-16.

[3] Carr BG, Goyal M, Band RA, Gaieski DF, Abella BS, Merchant $\mathrm{RM}$, et al. A national analysis of the relationship between hospital factors and post-cardiac arrest mortality. Intensive Care Med 2009;35;505-11.

[4] Fishman GI, Chugh SS, DiMarco JP, Albert CM, Anderson ME, Bonow RO, et al. Sudden cardiac death prediction and prevention: report from a National Heart, Lung, and Blood Institute and Heart Rhythm Society Workshop. Circulation 2010;122;2335-48.

[5] Sibai BM, Mabie BC, Harvey CJ, Gonzalez AR. Pulmonary edema in severe preeclampsia-eclampsia: analysis of thirty-seven consecutive cases. Am J Obstet Gynecol 1987;156;1174-9.
[6] Easterling TR, Benedetti TJ. Pre-eclampsia: a hyperdynamic disease model. Am J Obstet Gynecol 1989;160;1447-53.

[7] Garg RK, Kumar N, Malhotra HS. Posterior reversible encephalopathy syndrome in eclampsia. Neurol India 2018;66; 1316-23.

[8] Fisher N, Saraf S, Egbert N, Homel P, Stein EG, Minkoff H. Clinical correlates of posterior reversible encephalopathy syndrome in pregnancy. J Clin Hypertens 2016;18;522-7.

[9] Mijangos-Méndez JC, Aguirre-Avalos G, Corona-Jimenez F, Ortiz-Macias IX, López-Pulgarín JA, Chavez-Peña Q, et al. Cerebral vasospasm in cortical blindness associated with preeclamsia/eclampsia syndrome. Gynecol Obstet 2017;7; 1000454

[10] Shaikh N, Nawaz S, Chanda A, Nahid S, Zubair M, Ummunnisa F. Posteclampsia sudden cardiac arrest (SCA): a rare etiology. Case Rep Obstet Gynecol 2020;2020;8862839.

[11] Swartjes JM, Schutte MF, Bleker OP. Management of eclampsia: cardiopulmonary arrest resulting from magnesium sulfate overdose. Eur J Obstet Gynecol Reprod Biol 1992;47;73-5.

[12] Wex F, Luze R, Franke J, Ramoni A, Gizewski E, Lucovnik M, et al. Magnesium sulfate overdose resulting in maternal cardiac arrest: a case report. Clin Obstet Gynecol Reprod Med 2020; $6 ; 1-4$.

[13] Seo MH, Sung WY. A case of near-sudden unexpected death in epilepsy due to ventricular fibrillation. Open Access Emerg Med 2019;11;161-6.

[14] Kubota-Sjogren Y, Nelson-Piercy C. Fulminant antenatal pulmonary oedema in a woman with hypertension and superimposed preeclampsia. BMJ Case Rep 2015;2015;bcr2015212751.

[15] van der Lende M, Surges R, Sander JW, Thijs RD. Cardiac arrhythmias during or after epileptic seizures. J Neurol Neurosurg Psychiatry 2015;87;69-74. 